\title{
Nonlinear Analysis of BOLD Signal: Biophysical Modeling, Physiological States, and Functional Activation
}

\author{
Zhenghui $\mathrm{Hu}$ and Pengcheng Shi \\ Medical Image Computing Group \\ Hong Kong University of Science and Technology \\ Clear Water Bay, Kowloon, Hong Kong \\ \{eezhhu, eeship\}@ust.hk
}

\begin{abstract}
There is an increasing interest in exploiting the biophysical plausible models to investigate the physiological mechanisms that underlie observed BOLD response. However, most existing studies do not produce reliable model parameter estimates, are not robust due to the linearization of the nonlinear model, and do not perform statistics test to detect functional activation. To overcome these limitations, we developed a general framework for the analysis of fMRI data based on nonlinear physiological models. It performs system dynamics analysis to gain meaningful insight, followed by global sensitivity analysis for model reduction which leads to better system identifiability. Subsequently, a nonlinear filter is used to simultaneously estimate the state and parameter of the dynamic system, and statistics test is performed to derive activation maps based on such model. Furthermore, we investigate the change of the activation maps of these hidden physiological variables with experimental paradigm through time as well.
\end{abstract}

\section{Introduction}

A major problem in the interpretation of the fMRI BOLD signal is that the measurements are only indirectly related to the neural activity and interregional interactions from which they derive. Most current approaches to fMRI analysis uses linear convolution models that relate experimentally designed inputs, through an empirical hemodynamic response function (hrf), to observed BOLD signals. Such approaches, however, are blind to the mechanisms that underlie physiological changes, while it is important to have a quantitative understanding of those factors that are more directly related to the neural activity, such as changes in flow, oxygen extraction, blood volumes and their combined effects. These physically meaningful measures are needed to clarify the relationship between neural activation and experimental paradigm, and the significance of the observed transients in the BOLD signal.

The Balloon Model has been developed as a comprehensive biophysical model of hemodynamic modulation, and provide a possible platform to understand the changes of physiological variables during brain activation 1 1 . It combines 
the coupling mechanism of manifold physiological variables, and has successfully simulated pronounced transients in BOLD signal, including initial dips, overshoots and a prolonged post-stimulus undershoot. This model has been extended to include the relationship between evoked neural activity and blood flow [2], and transformed into to a nonlinear state space representation [3].

There have been several attempts applying such a biophysical model to the analysis of fMRI data, with some limitations. First, typical fMRI response for brief neuronal events lasts $12-16 \mathrm{~s}$ (with 6-8 observations), while the balloon model includes six unknown constant hemodynamic parameters and a neural parameter varying to trial. It is thus difficult to estimate all seven variables at the same time ( at worst, the model can be underdetermined if one has few than seven image volumes. ). Thus, it is of great benefit to reasonably reduce the number of parameters needed to be estimated in order to increase the system identifiability. Some of the existing efforts discard such concern, and attempt to estimate all parameters [2 4] 3, while others try to reduce the parameters through a regional linear analysis [5] or arbitrary assignment [6. Secondly, The hrf typically possesses strong nonlinear characteristics. The linearized approximation method [2] 4] 3] 5] only reliable when time scale is discretized to sufficiently small that system are almost linear, and it can result in nonstable estimates, if the assumptions of local linearity is violated. Thus, a nonlinear estimation algorithm should be considered as a natural choices to against approaches of linearized approximation. Thirdly, Neuroimaging had been concerned predominantly with the localization of function. Most existing studies assume that the locus of a functional activation is known, which is determined by other ways. The estimation is then limited in a known activated voxel, not build a statistic to detect activation [2] 3] 6].

To overcome these shortcomings, we present a general framework for using Balloon model in the analysis of fMRI data. Firstly, we propose a quantitative model reduction method, relying on global, variance-based sensitivity analysis (SA) method, to assess the relative importance of system parameters in Balloon model. Subsequently, we present a nonlinear filter approach for joint state and parameter estimation of nonlinear dynamics system and provide a corresponding statistical test to derive activation maps based on such model. Furthermore, attribute to such innovation estimation process, we provide a possible approach for detecting the change of the activation maps of these hidden physiological variables with experimental paradigm through time as well.

\section{Hemodynamics Balloon Model}

The Balloon model consists of three subsystem linkings: (1) neural activity to changes in flow; (2) changes in flow to changes in blood volume and venous outflow; (3) changes in flow, volume and oxygen extraction fraction to changes in deoxyhemoglobin $(\mathrm{dHb})$. It describes the dynamics intertwinement between 
the blood flow $f$, the blood venous volume $v$ and the veins $\mathrm{dHb}$ content $q$, can be given as the following [1]:

$$
\left\{\begin{array}{l}
\ddot{f}=\epsilon u(t)-\frac{\dot{f}}{\tau_{s}}-\frac{f-1}{\tau_{f}} \\
\dot{v}=\frac{1}{\tau_{0}}\left(f-v^{1 / \alpha}\right) \\
\dot{q}=\frac{1}{\tau_{0}}\left(f \frac{1-\left(1-E_{0}\right)^{1 / f}}{E_{0}}-v^{1 / \alpha} \frac{q}{v}\right)
\end{array}\right.
$$

where $\epsilon$ is neuronal efficacy; $u(t)$ is the neuronal inputs; $\tau_{s}$ reflects signal decay; $\tau_{f}$ is the feedback autoregulation time constant; $\tau_{0}$ is the transit time; $\alpha$ is the stiffness parameter; and $E_{0}$ represent the resting oxygen extraction fraction. All variables are expressed in normalized form, relative to resting values. Eq. (11) has a second-order time derivative, and we can write this system as a set of four first-order ODEs by introducing a new variable $s=\dot{f}$.

Furthermore, the BOLD signal can be expressed as:

$$
\left\{\begin{array}{l}
y(t)=V_{0}\left(k_{1}(1-q)+k_{2}\left(1-\frac{q}{v}\right)+k_{3}(1-v)\right), \\
k_{1}=7 E_{0}, \quad k_{2}=2, \quad k_{3}=2 E_{0}-0.2,
\end{array}\right.
$$

appropriate for a 1.5 Tesla magnet [1, where $V_{0}$ is the resting blood volume fraction.

Statistical models usually can be explained as the fixed effects, which capture the underlying pattern, plus the error term. Thus, we rewrite Eqs. (11), (2) as:

$$
\begin{aligned}
& \dot{\mathbf{x}}=f(\mathbf{x}, \boldsymbol{\beta}, \mathbf{u}, \mathbf{v}), \quad v \sim N(0, \mathbf{Q}) \\
& \mathbf{y}=h(\mathbf{x}, \boldsymbol{\beta}, \mathbf{w}) \quad w \sim N(0, \mathbf{R})
\end{aligned}
$$

where $f$ and $h$ are nonlinear equations, $\mathbf{x}(t)=[s(\dot{f}), f, v, q]^{T}$ is the state of the system, $\boldsymbol{\beta}=\left\{\epsilon, \tau_{s}, \tau_{f}, \tau_{0}, \alpha, E_{0}, V_{0}\right\} \in \mathbb{R}^{l}$ is system parameters, the neuronal inputs $\mathbf{u}$ represents system input, $\mathbf{v}$ is the noise process caused by disturbances and modeling errors, $\mathbf{y}$ is the observation vector, and $\mathbf{w}$ is measurement noise. Eqs (3) and (4) constitute a state-space representation of fMRI BOLD responses to given stimulation

\section{Dynamics in State Space}

A state space $\left(\mathbf{x}(t)=[s(\dot{f}), f, v, q]^{T}\right)$ and a rule (Eqs. 11) for following the evolution of trajectories starting at various initial conditions constitute a dynamical system. It is interesting to build some intuition for its dynamics.

The fixed point of the system evolution can be found, by setting the four time derivatives $\left.\dot{\mathbf{x}}\right|_{x=x_{0}}=f\left(\mathbf{x}_{0}\right)=0$. Thus, we have a equilibrium state, $\mathbf{x}_{0}=$ $\left[0, \epsilon \tau_{f} u_{0}+1,\left(\epsilon \tau_{f} u_{0}+1\right)^{\alpha},\left(\epsilon \tau_{f} u_{0}+1\right)^{\alpha} \frac{1-\left(1-E_{0}\right)^{1 /\left(\epsilon \tau_{f} u_{0}+1\right)}}{E_{0}}\right]^{T}$. The nature of the fixed point is determined by the characteristic values of the Jacobian matrix of 

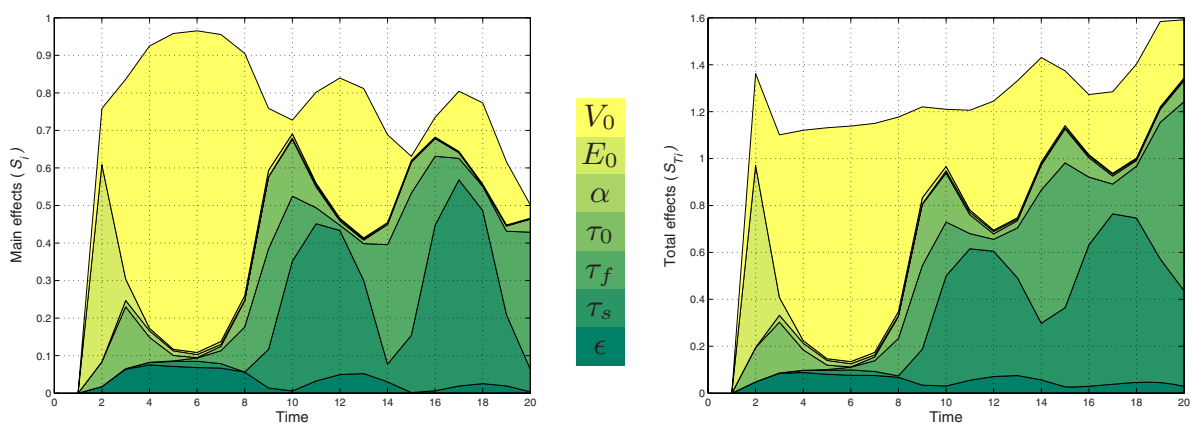

Fig. 1. Area plot of the main effects (left) and total effects (right) sensitivity indices (obtained with $N(d+2)$ simulations, where $N=200, d$ is the dimensionality of parameters) to a $2 s$ stimulated stimulus for the balloon model

partial derivatives evaluated at the fixed point. The Jacobian matrix for the set of equation is defined as:

$$
\mathbf{J}=\left(\begin{array}{cccc}
-\frac{1}{\tau_{s}} & -\frac{1}{\tau_{f}} & 0 & 0 \\
1 & 0 & 0 & 0 \\
0 & \frac{1}{\tau_{0}} & -\frac{v^{1 / \alpha-1}}{\alpha \tau_{0}} & 0 \\
0 & \frac{1}{\tau_{0}}\left(\frac{1-\left(1-E_{0}\right)^{1 / f}}{E_{0}}-\frac{\left(1-E_{0}\right)^{1 / f} \ln \left(1-E_{0}\right)}{E_{0} f}\right) \frac{q}{\tau_{0}}\left(1-\frac{1}{\alpha}\right) v^{1 / \alpha-2}-\frac{v^{1 / \alpha-1}}{\tau_{0}}
\end{array}\right)
$$

Its eigenvalues evaluated $\lambda$ at the fixed point $\mathbf{x}_{0}$ are:

$$
\left\{\frac{-\frac{1}{\tau_{s}}+\sqrt{\frac{1}{\tau_{s}^{2}}-\frac{4}{\tau_{f}}}}{2}, \frac{-\frac{1}{\tau_{s}}-\sqrt{\frac{1}{\tau_{s}^{2}}-\frac{4}{\tau_{f}}}}{2},-\frac{\left(\epsilon \tau_{f} u_{0}+1\right)^{1-\alpha}}{\alpha \tau_{0}},-\frac{\left(\epsilon \tau_{f} u_{0}+1\right)^{1-\alpha}}{\tau_{0}}\right\}
$$

All $\lambda$ are negative, dictates the volumes contract along all directions of the coordinates of the phase space, and mean that the volume will shrink to a point in time. Since the sum of the eigenvalues, $\operatorname{Tr}(J)=\lambda_{1}+\lambda_{2}+\lambda_{3}+\lambda_{4}<0$, it is a dissipative system. The system has not any long-terms dynamic behavior.

\section{Model Reduction}

We want to investigate the contribution of parameter factor to the output variation of model. A parameter that does not contribute to the output variance neither singular nor in combination with other parameters can be frozen to any value within its range of variation. This direct us to sensitivity analysis (SA). SA study how the uncertainty in the output of a model can be apportioned to different sources of uncertainty in the model input [7, and is consider as a imperative [8] or recommendation [9] in any field where models are used. 
Firstly, we introduce a global, variance-based SA method [7]. The total output variance $V(Y)$ for a model with $d$ parameters can be decomposed as:

$$
V(Y)=\sum_{i} V_{i}+\sum_{i<j} V_{i j}+\sum_{i<j<k} V_{i j k}+\cdots+V_{12 \ldots d}
$$

where $V_{i}=V\left(E\left(Y \mid X_{i}\right)\right), V_{i j}=V\left(E\left(Y \mid X_{i}, X_{j}\right)\right)-V_{i}-V_{j}$ and so on. They called partial variances and are orthogonal each other. Then, main effects terms for parameter $\beta_{i}$ can define as:

$$
S_{i}=\frac{V\left[E\left(Y \mid \beta_{i}\right)\right]}{V(Y)}
$$

This measure indicates the relative importance of an individual parameter $\beta_{i}$, in driving the uncertainty. Total total effects terms can be defined as:

$$
S_{T i}=\frac{E\left[V\left(Y \mid \beta_{-i}\right)\right]}{V(Y)}
$$

$\beta_{-i}$ are all the parameters except $\beta_{i}$. This measure indicates the sum of all terms in the variance decomposition that include $\beta_{i}$.

Thus, given input $u(t)$, an typical parameter set $\beta=\{0.54,1.54,2.46,0.98$, $0.33,0.34,0.02\}$ and their uncertainty range $\sigma^{2}=\left\{0.1^{2}, 0.25^{2}, 0.25^{2}, 0.25^{2}, 0.045^{2}\right.$, $\left.0.1^{2}, 0.005^{2}\right\}$ [2], we exploit SA for model simplification.

The area plot for the main indices is shown on left in Figure1. The parameter with high first-order effects are $V_{0}$ and $\tau_{f}$. A high value for $S_{i}$ give a consistent contribution to the model output variance and indicates a good candidate for output uncertainty reduction through new research. Furthermore, we also display the total indices, $S_{T i}$, on right in Figure 1. This measure indicates the total contribution to the variance of $Y$ due to $\beta_{i}$ singularly or combination with others. Thus it can be employed to identify unessential parameters. We can see that the parameter $\alpha$ are non-influential at any time point. It can be frozen to any value within its range of uncertainty. Therefore we assume that $\alpha=0.33$ in the following parameters estimation.

Furthermore, it should be noted that the simultaneous estimation to parameter $V_{0}$ and other factors would be impossible, but only their product (see Eq. 2]). Thus, we impose a physiological plausible value $V_{0}=0.02$ throughout brain.

\section{Nonlinear System Identification}

We addressed this nonlinear state-space estimation (Eqs. 3, 4) using unscented kalman filter (UKF) [10] to maintain the nonlinearities present in the biophysical model. UKF propagates variables mean and covariance through the unscented transformation (UT), and is high accuracy and robustness for nonlinear models.

UT deterministically chose a set of weighted sigma points so that first two moments of these points match the prior distribution, and propagates them through the actual nonlinear function. Then, the properties of the transformed 
set can be recalculated from these propagated points. It can capture the posterior mean and covariance accurately to the 3rd order (Taylor series expansion) for any nonlinearity. The standard UKF implementation for state-estimation can be find in 11. In the joint filtering approach (state estimate and parameter identification), the hidden system state and parameters are concatenated into a single higher-dimensional joint state vector, that is joint state vector $\mathbf{x}=$ $\left\{\dot{f}, f, v, q, \epsilon, \tau_{s}, \tau_{f}, \tau_{0}, E_{0}\right\}^{T}$ in here, then, a standard UKF is now run on the joint state space to produce simultaneous estimates of the states and the parameters.

Since the differential equations in Eq3. are not soluble analytically, we employ a fourth order Runge-Kutta method to investigate the information about the trajectory, where step length $h$ set as $0.2 s$ to make the truncation error involved sufficiently small. Furthermore, while the initial input $u_{0}=1$ (section 7 ), the state necessarily converge to their equilibrium points $\mathbf{x}_{0}=\left[0, \epsilon \tau_{f}+1,\left(\epsilon \tau_{f}+\right.\right.$ $\left.1)^{\alpha}, \frac{1-\left(1-E_{0}\right)^{1 /\left(\epsilon \tau_{f}+1\right)}}{E_{0}}\left(\epsilon \tau_{f}+1\right)^{\alpha}\right]^{T}$ (Sec 3) , thus the initial condition was set as $\mathbf{x}(0)=[0,2.328,1.322,0.635,0.54,1.54,2.46,0.98,0.34]^{T}$.

\section{Statistical Test}

We want to establish a statistical test to detect activations voxelwise. It seem is difficult to establish probabilities on individual parameters in such a nonlinear case, since single factor effects can not be partitioned due to the nonlinear correlation between parameters. However, we can assess the nonlinear model hypotheses, and compare models in a hierarchy, where inference is based on a $F$-statistic.

Suppose we have a nonlinear model with parameter $\boldsymbol{\beta}$, and we wish test $\mathcal{H}: h(\mathbf{x}, \boldsymbol{\beta})=0$. The full model is $\mathbf{y}=h(\mathbf{x}, \boldsymbol{\beta})+\mathbf{w}$, which reduce to the reduced model: $\mathbf{y}=$ const $+\mathbf{w}$, when $\mathcal{H}$ is true. Denote the residual sum-of-squares for the full and reduced models by $S_{f}$ and $S_{r}$ respectively. Under $\mathcal{H}, S_{f}$ and $S_{r}$ are independent, $S_{f} \sim \chi_{p}^{2}$ and $S_{r} \sim \chi_{q}^{2}$. The following $F$-statistic expresses evidence $\mathcal{H}$ versus the alternative $h \neq 0$ :

$$
F=\frac{\frac{S_{f}}{p}}{\frac{S_{r}}{q}} \sim F_{p, q}
$$

that is the random variable $F$ has Snedecor's $F$ distribution with $p$ and $q$ degrees of freedom. The larger $F$ gets the more unlikely it is that $F$ was sampled under the null hypothesis $\mathcal{H}$. Significance can then be assessed by comparing this statistic with the appropriate $F$-distribution.

\section{Experiment}

2 distinct texture photograph were presented in $2 s$ for touch perception followed by a $14 s$ rest, staring with stimulus. Total 96 acquisitions were made $(\mathrm{RT}=2 s)$, in periods of $16 \mathrm{~s}$, giving 1216 -second circles. We chose the largest activation blob using SPM2, as region of interest. 


\section{Result and Discussion}

Figure (2). shows the estimated behavior of the state functions of the hemodynamic approach for the largest activation blob in SPM2. All these predictions of
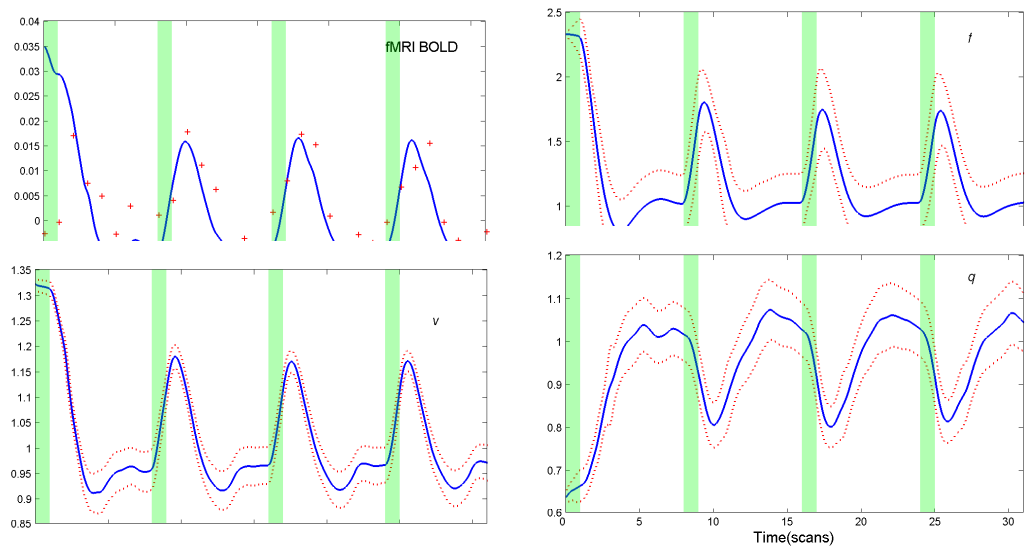

Fig. 2. The time series of the estimated states functions of hemodynamic response to touch figure perception tasks. (a-d): BOLD signal $y$ (the measured signal (red plus sign) and the filtering process (blue line)), the blood flow $f$, the blood venous volume $v$ and the veins $\mathrm{dHb}$ content $q$. Each stimulus event, which was simulated by rectangular pulse of width $2 s$, is shown as strips in green. These red bounds are given by the square root of the diagonals of the covariance matrix.
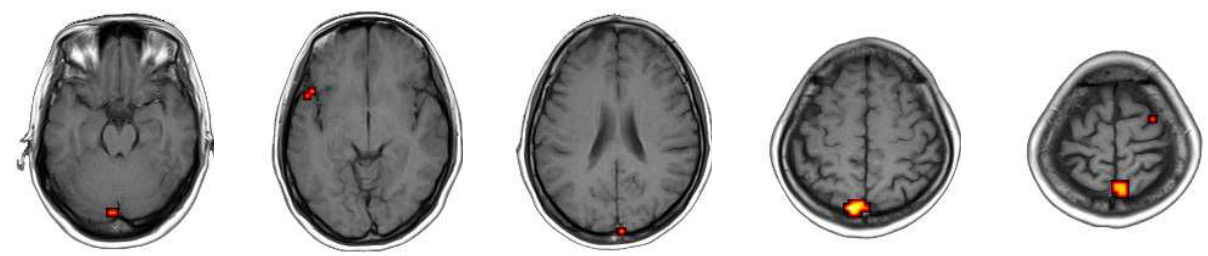

Balloon model
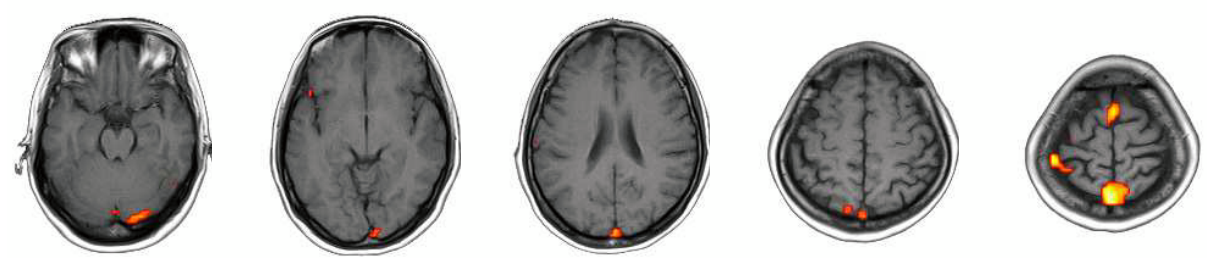

GLM

Fig. 3. Activation maps obtained with balloon model and GLM $(p<0.001)$ 
the Balloon model concur with the known physiological effects in fMRI BOLD signal. The values of these parameters all are in the range of previously report 2. 3. These physiological plausible parameter estimated may provide valuable information to evaluate activation.

The same joint estimation approach then deal with all voxels throughout the brain in a voxel-specific fashion to investigate the localization of function, that is, where in the brain mediate a cognitive process of interest. Figure 3 shows the resulting activation detection overlaying on anatomical image for balloon model and general linear model (GLM). Two methods yielded the similar activation map, however, have different maximum activation.

In this paper, we developed a general framework for using physiological models in the analysis of fMRI data. It includes dynamical analysis of system, model reduction, system identification and activation detection. The method is also efficient to deal with the fMRI data in others physiological model and experimental paradigms, including block-design and event-related.

\section{References}

1. Buxton, R.B., Wong, E.C., Frank, L.R.: Dynamics of blood flow and oxygenation changes during brain activation: The balloon model. Magn. Reson. Med. 39, 855864 (1998)

2. Friston, K.J., Mechelli, A., Turner, R., Price, C.J.: Nonlinear responses in fmri: The balloon model, volterra kernels, and other hemodynamics. NeuroImage 12, 466-477 (2000)

3. Riera, J.J., Watanabe, J., Kazuki, I., Naoki, M., Aubert, E., Ozaki, T., Kawashima, R.: A state-space model of the hemodynamic approach: nonlinear filtering of bold signals. NeuroImage 21, 547-567 (2004)

4. Friston, K.J.: Nonlinear responses in fmri: Bayesian estimation of dynamical systems: An application to fmri. NeuroImage 16, 513-530 (2002)

5. Deneux, T., Faugeras, O.: Using nonlinear models in fmri data analysis: model selection and activation detection. Neuroimage 32, 1669-1689 (2006)

6. Johnston, L.A., Duff, E., Egan, G.F.: Partical filtering for nonlinear bold signal analysis. In: Larsen, R., Nielsen, M., Sporring, J. (eds.) MICCAI 2006. LNCS, vol. 4190, pp. 292-299. Springer, Heidelberg (2006)

7. Saltelli, A., Ratto, M., Tarantola, S., Campolongo, F.: Sensitivity analysis practices: strategies for model-based inference. Reliability Engineering \& System Safety 91, 1109-1125 (2006)

8. EC: European commission's communication on extended impact assessment brussels, com (2002) 276 final. IEEE (05/06/2002), http://europa.eu.int/comm/governance/docs/index_en.htm

9. EPA: The us environmental protection agency science policy council, white paper on the nature and scope of issues on adoption of model use acceptability guidance IEEE, http://www .epa.gov/osp/crem/library/whitepaper_1999.pdf .

10. Julier, S.J., Uhlmann, J.K.: Unscented filtering and nonlinear estimation. Proceeding of The IEEE 92, 401-422 (2004)

11. Merwe, V., Wan, E.R.: The square-root unscented kalman filter for state andparameter-estimation. In: ICASSP. vol. 6, pp. 3461-3464 (2001) 